American Journal of Biochemistry and Biotechnology 3 (3): 145-149, 2007

ISSN 1553-3468

(C) 2007 Science Publications

\title{
Biomedicine and Informatics Model of Alzheimer's Disease
}

\author{
Kang Cheng and Chang-Hua Zou \\ Science Research, 205 Hana Road, Edison NJ 08817,USA
}

\begin{abstract}
In a perspective of biomedicine and informatics, the mechanism of Alzheimer's, senile amnesia, or other aging-associated and cognitive impairment related diseases involve four important informative processing procedures: propagation, consolidation, retrieval and cognition, In this study, we systematically model the four procedures based on published experimental data. When modeling the propagation, we develop an equivalent circuit of biological membrane to describe how the neuron signals are propagated, attenuated, compensated, transferred, oscillated and filtered; and how wrong signals are related to the diseases. Our circuit involves complex admittances, resonance angular frequencies, propagating constants, active pump currents, transfer functions in frequency domain and memory functions in time domain. Our circuit explains recurrent of brain neurons and clinical EEG frequencies as well as represents an encoding of current or electric field intensity (EFI). When modeling the consolidation and the retrieval of long term memory (LTM), we emphasize the EFI consists of a non conservative electric field intensity (NCEFI) and a conservative electric field intensity (CEFI). It is mostly a NCEFI of acquired information to evoke an informative flow: from the inherited or mutant DNA to the transcribed RNA, from the transcribed RNA to the translated proteins. Some new synthesized proteins relate to the memory functions. The charges of the proteins and the memory functions mostly store the LTM and play an important role during the LTM retrieval. When modeling the cognition in working memory (WM), our model demonstrates: if a sum of two sets of EFI signals is enhanced positively (or negatively), at a sub-cellular level (especially at the axon hillock), the sum supports a positive (or negative) cognition; otherwise, the sum tends to be no cognition. A set of related brain neurons in WM work organically to vote, by EFI signal outputs through their axons, if they recognize or cognize an object.
\end{abstract}

Key words: DNA, RNA, protein, cognition, impairment, memory, working, long, term, retrieval

\section{INTRODUCTION}

Alzheimer's disease is an aging-related progressive neurodegenerative disorder ${ }^{[1]}$. It is the most common form of the dementia and it affects about $5 \%$ of adults over 65 years $\operatorname{old}^{[2]}$. The prevalence doubles approximately every 5 years beyond age $65^{[3]}$. Some study suggests that nearly half of the people aged 85 years and older suffer from this devastating disorder ${ }^{[1,4]}$.

On November 3, 1906, Dr. Alzheimer reported his clinical and pathological findings, under the title of "On a peculiar disease process of the cerebral cortex", at a conference in Tübingen. In his presentation, Dr. Alzheimer discussed cognitive and non-cognitive deficits of his famous patient Auguste D and reported that on post-mortem he found argyrophilic plaques, tangles and atherosclerotic changes in the patient's brain $^{[5,6]}$. In 1910, the term of Alzheimer's disease was firstly used to describe the disease that Auguste D $\operatorname{had}^{[6,7]}$. The syndromes of Alzheimer's disease are disturbances of cognitive functions including memory, judgment, decision-making, orientation to physical surroundings and language ${ }^{[8]}$. The characteristic neuropathological hallmarks of Alzheimer's disease are the extra cellular deposition of a 39-43 amino acid protein termed $\beta$-amyloid (or $\beta \mathrm{A}$ ), in the cerebrovasculature (1) and cores of senile plaques (2), as well as the formation of paired helical filaments (PHFs) that comprise intracellular neurofibrillary tangles (NFTs) (3), neuropil threads and senile plaque neurites. The principle component of PHFs is an abnormally hyperphosphorylated form of the microtubule-associated protein tau (4). Other features of Alzheimer's disease pathology include neuronal and synaptic fallout that disrupts neurotransmission to the neocortex, as well as excitatory amino acidergic pyramidal neurons ${ }^{[9]}$. 
It is now 101 years since nosological definition of Alzheimer's disease emerged ${ }^{[10]}$. Amnestic mild cognitive impairment (MCI) is a relative newly defined clinical entity that requires memory decline while activities of daily living remain intact. Most Amnestic MCI patients develop Alzheimer's disease ${ }^{[11]}$. In addition, evidence continues to accumulate that agingassociated changes in several neuroendocrine system are involved in important but complex way in the pathophysiology of the late behavioral changes and disorders ${ }^{[12]}$.

In a perspective of biomedicine and informatics ${ }^{[13-}$ ${ }^{14]}$, the mechanism of Alzheimer's, senile amnesia, or other aging-associated and cognitive impairment related diseases involve four important informative processing procedures: propagation, consolidation, retrieval and cognition, In this study, we systematically model the four procedures based on published experimental data, at a level of molecule, cell and sub-organ. To our knowledge, there are not published models that are the same as or similar to ours.

\section{MATERIALS AND METHODS}

Modeling informative propagation of neuron membrane: Experimental data have confirmed, neuron channels quasi-periodically open and close, neuron voltage signals propagate by jumping and quasi frequency encoding along the axon membrane or by diffusing in the cellular plasma, active pumps cost biochemical energy to keep a balance of cellular ions concentration and a rest voltage across the membrane $e^{[15-16]}$.

There have been many electric circuit models of biological membrane based on both classic theory ${ }^{[15-16]}$ and quantum mechanics ${ }^{[17-18]}$, but no inductors, no tangential resistors and capacitors, nor active pumps have been included in the models. Additionally, there is not any model to completely describe how the neuron signals are propagated, attenuated, compensated, transferred, oscillated and filtered; and how the wrong signals are related to the diseases, in a view of biomedicine and informatics. Our model could overcome these shortcomings of the previous models.

Quasi periodical open and close of neuron ions channels cause a working angular frequency $\omega$ of electromagnetic signal across or along a neuron membrane. Figure 1 illustrates an equivalent circuit of a neuron membrane patch for our neural network model, based on published experimental data ${ }^{[16]}$. The size of a neuron membrane patch may be approximately that of a synapse or that of a length between two nodes of Ranvier on an axon Fig. 2.
When considering neuron signals propagate along an axon (or a dendrite) membrane at a time t Fig. 1, we assume a position $\mathrm{i}$ is in a $\mathrm{z}$ coordinate; an active pump current $\mathrm{I}_{\mathrm{a}, \mathrm{i}}(\mathrm{t})$ keeps a rest membrane voltage; a normal voltage $\mathrm{V}_{\mathrm{n}, \mathrm{i}}(\mathrm{t}, \mathrm{z})$ applies to a resistor $\mathrm{R}_{\mathrm{n}, \mathrm{i}}$ and an inductor $\mathrm{L}_{\mathrm{n}, \mathrm{i}}$ or a capacitor $\mathrm{C}_{\mathrm{n}, \mathrm{i}}$, the resistor and the inductor are varying depending on if the channels open or close; a tangential current $\mathrm{I}_{\mathrm{t}, \mathrm{i}}(\mathrm{t}, \mathrm{z})$ flows through a resistor $\mathrm{R}_{\mathrm{t}, \mathrm{i}}$ and an inductor $\mathrm{L}_{\mathrm{t}, \mathrm{i}}$ or a capacitor $\mathrm{C}_{\mathrm{t}, \mathrm{i}}$. Appling Kirchhoff's law with boundary conditions, we can obtain,

$$
\text { inside of a cell }
$$

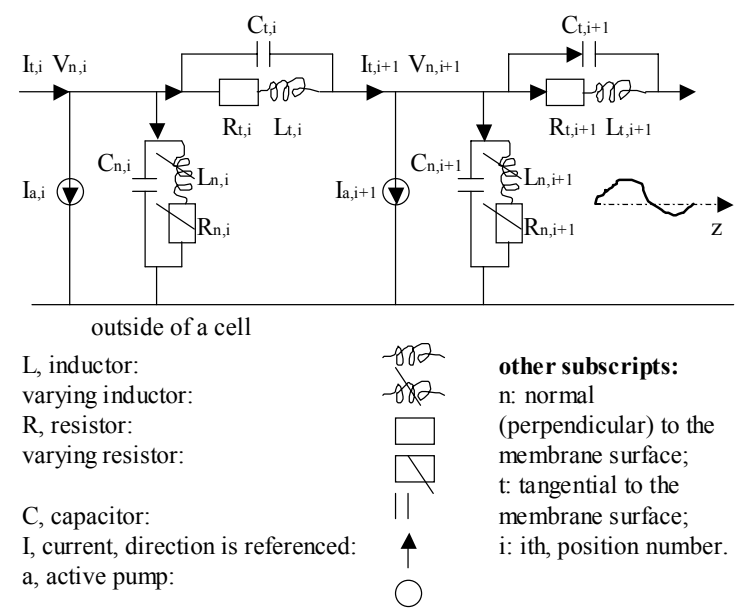

Fig. 1:An equivalent circuit of a neuron membrane patch in our neural network model. Published models do not have $\mathrm{I}_{\mathrm{a}, \mathrm{i}}, \mathrm{C}_{\mathrm{t}, \mathrm{i}}, \mathrm{R}_{\mathrm{t}, \mathrm{i}}, \mathrm{L}_{\mathrm{t}, \mathrm{i}}, \mathrm{L}_{\mathrm{n}, \mathrm{i}}$. See neuron membrane patches in Fig. 2

$V_{n, i}(t, z)=V_{n, i}(t) \exp \left(-\gamma_{i} z\right)+I_{a, i}(t) / Y_{n, i}(\omega)$

$I_{t, i}(t, z)=-V_{n, i}(t, z) \gamma_{i} Y_{t, i}(\omega)$

Where, $V_{n, i}(t) \propto, \exp ((-\alpha-j \omega) t)$ based on experimental data $^{[16]}, \alpha$ is a temporal attenuation constant; we define $\mathrm{Y}_{\mathrm{n}, \mathrm{i}}(\omega)$ and $\omega_{\mathrm{n}, \mathrm{i}}$ as a normal admittance and a normal resonance angular frequency respectively:

$$
\begin{aligned}
& Y_{n, i}(\omega)=1 /\left(R_{n, i}+j \omega L_{n, i}\right)+j \omega C_{n, i} \\
& \omega_{n, i}=\sqrt{\frac{1-\left(C_{n, i} / L_{n, i}\right) R_{n, i}^{2}}{C_{n, i} L_{n, i}}}
\end{aligned}
$$

and we also respectively define $\mathrm{Y}_{\mathrm{t}, \mathrm{i}}(\omega)$ and $\omega_{\mathrm{t}, \mathrm{i}}$ as a tangential admittance and a tangential resonance angular frequency that have the same forms as equations (3) and (4), but use subscript $t$ instead of $n$. $\gamma_{\mathrm{i}}=\left(\mathrm{Y}_{\mathrm{n}, \mathrm{i}}(\omega) / \mathrm{Y}_{\mathrm{t}, \mathrm{i}}(\omega)\right)^{1 / 2}$ is a propagating constant. 


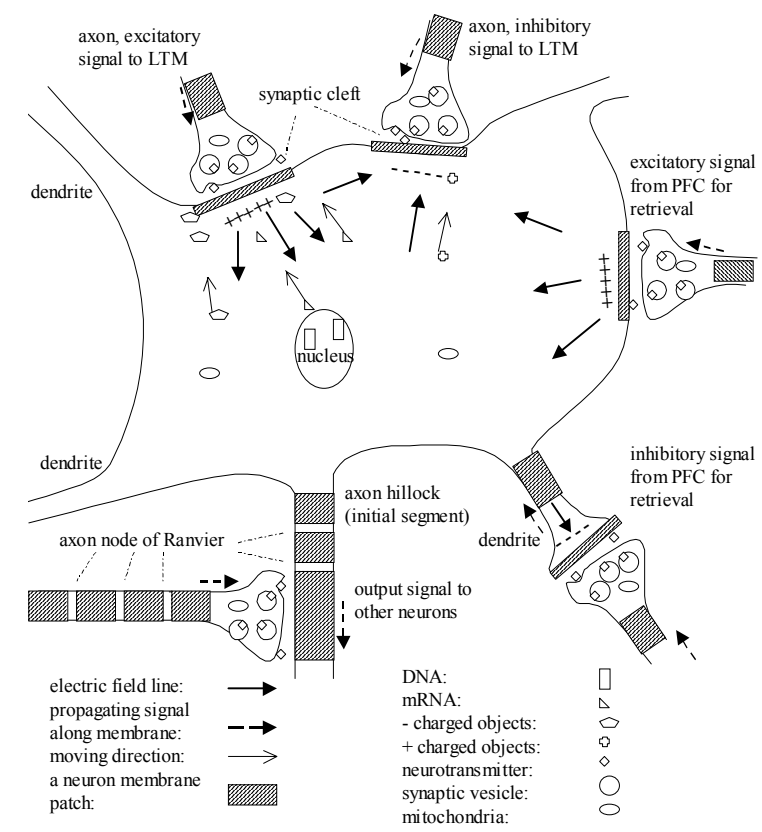

Fig. 2: A neuron for visual LTM is in medial or interior cortex. DNAs store inherited (or mutant) information, mRNAs store transcribed information and proteins (e.g. F-actin, Arc) store acquired (translated) information, i.e. LTM. An equivalent circuit of a membrane patch is shown in Fig. 1. The draw is not in scale

When considering a membrane patch at a post synapse Fig. 2, we ignore tangential $\mathrm{C}_{\mathrm{t}, \mathrm{i}}, \mathrm{L}_{\mathrm{t}, \mathrm{i}}, \mathrm{R}_{\mathrm{t}, \mathrm{i}}$ and use only normal $C_{n, i} L_{n, i}$ and $R_{n, i}$ to construct an equivalent circuit, because the currents mostly penetrate the membrane rather than propagate along the membrane ${ }^{[16]}$. A normal transfer function $\mathrm{H}_{\mathrm{n}, \mathrm{i}}(\omega)$ equals to a normal admittance $\mathrm{Y}_{\mathrm{n}, \mathrm{i}}(\omega)$. We believe the transfer function of a synapse relates to a memory unit, because it relates to a memory function in time domain ${ }^{[19]}$.

Equations from (1) to (4) could explain spontaneous electromagnetic oscillations, recurrent of brain neurons and clinical EEG frequencies as well as represent a frequency encoding ${ }^{[16]}$, a filter effect and a wave informative propagation along neuron membrane. The spatial signal attenuation during the propagation is compensated by the channel currents of the next patch at position $\mathrm{i}+1$ in $\mathrm{z}$ coordinate.

Modeling informative consolidation and retrieval of long term memory (LTM): Environmentally acquired information must be consolidated or stored as LTM to be retrieved later. Published experimental data have suggested or confirmed that visual LTM is stored in the medial and interior temporal cortex neurons ${ }^{[20-21]}$, a frequent stimulation with same signals may produce a long term potentiation (LTP) and a LTM consolidation is performed under a condition of LTP and involves new structures of brain neurons ${ }^{[22-23]}$. The new structures could be constructed with F-actins ${ }^{[24]}$, activity-regulated cytoskeleton ${ }^{[25]}$, RNAs and related proteins $^{[26]}$, AMPA-receptors ${ }^{[27]}$ or Trk receptors ${ }^{[28]}$. Some significant biochemical models have developed to elucidate the mechanisms of process of LTP and $\mathrm{LTM}^{[20-21]}$. However, the questions what kind of force drives RNAs or proteins to move, where and how the LTM is consolidated and how the LTM is retrieved have not been answered in a perspective of biomedicine and informatics. Our model, based on the above experimental data, could answer the questions.

Our equation (2) also represents a current encoding. A current relates to a non conservative electric field intensity (NCEFI). Therefore, in a neuron body (see our model in Fig. 2, environmentally acquired information (signals) may be encoded into NCEFI by Ohm's law,

$\operatorname{NCEFI}(r, t)=\sum_{i} J_{i}(r, t) / \sigma(r, t)$

Where, $r$ is a position vector and $\mathrm{t}$ is a time parameter ${ }^{[14]}, J_{i}(r, t)$ is a vector of current density contributed directly or indirectly by ith synapse and relates to the propagating constants, the transfer (or memory) functions or the resonance frequencies of the neural network; and $\sigma(r, t)$ is a conductivity of the cellular plasma. During a LTM consolidation, the NCEFI triggers the inherited (or mutant) information (DNA) and evokes a RNA transcription; and drives some existed RNA and negatively charged objects to the excited post synapses or drives the positively charged objects to the inhibited post synapses. The new transcribed RNA is repulsed out of the nucleus by the $\mathrm{DNA}^{[13]}$ and then is driven to the excited post synapse mostly by the NCEFI. The protein (e.g. F-actin, Arc) translation around the excited post synapse occurs by guidance of the both existed and new transcribed RNA. Some of the new synthesized proteins may relate to the propagating constants, the transfer (or memory) functions or the resonance frequencies. The new translated proteins are usually charged. The charges and the memory functions mostly store the LTM. The charges have a conservative electric field intensity $(\operatorname{CEFI}(r, t))^{[14]}$. Applying Coulomb's law, we estimated $\operatorname{CEFI}(r, t)$ with a convolution of a total charge distribution in cellular plasma ${ }^{[14]}$. A total electric field intensity (EFI) in a neuron body should be:

$\operatorname{EFI}(r, t)=\operatorname{CEFI}(r, t)+\operatorname{NCEFI}(r, t)$ 
We believe the EFI plays an important role to build and to retrieve the LTM. When the LTM is retrieved, a force of EFI may open channels at an axon hillock and output signals to other neurons if it is beyond a threshold value. A neuron is an informative processing unit. No mater how to retrieve or to trigger a neuron that stores LTM, the neuron will produce a signal output corresponding to its LTM.

Modeling informative cognition in working memory (WM): Cognition or recognition is an informative process for both LTM and short term memory (STM). Published data have confirmed or suggested that a neuron for visual WM is in neocortex such as prefrontal cortex (PFC), perirhinal cortex, postrhinal cortex; the WM includes short-term representations of information that were recently experienced or retrieved from longterm representations of sensory stimuli. The WM activates the same dorsolateral prefrontal cortex neurons that: (a) maintained recently perceived visual stimuli; and (b) retrieved visual stimuli from $\mathrm{LTM}^{[21]}$. But, to our knowledge, the question how neurons work in WM has not been answered in a view of biomedicine and informatics. Our model could answer this question.

We use equations (5) and (6) to calculate the EFI signals in WM neuron bodies for the cognition. A diagram (not shown) of our model for the cognition in WM is similar to Fig. 2 in principle. Based the published experimental data ${ }^{[20-21]}$, our model demonstrates: if a sum of two sets of EFI signals (transmitted through synapses) is enhanced positively (or negatively), at a sub-cellular level (especially at the axon hillock), the sum supports a positive (or negative) cognition; otherwise, the sum tends to be no cognition $^{[14]}$. One set of signals can be from the recently experienced while another set can be from the LTM; or the both sets can be either from the recently experienced or from the LTM. A set of related brain neurons in WM work organically to vote, by EFI signal outputs through their axons, if they recognize or cognize an object.

\section{RESULTS AND DISCUSSION}

Based on our models, for patients who have Alzheimer's, senile amnesia, or other aging-associated and cognitive impairment related diseases, there could be the defected resistors, inductors, capacitors, or the abnormal permitivity, conductivity, charge storages, because of wrong inherited or transcribed information (DNA or RNA), $\beta$ A deposition, formation of PHFs (or NFTs), or damaged, degenerated, abnormally grown neurons. Therefore, the resonance frequencies, the memory functions or the propagating constants are unordinary, or the neuron informative propagation is broken or wrong functional, or the LTM is abnormal or nothing, or the WM has a poor or no cognition.

Our models in this study could also explain the mechanisms of other nerve system diseases, such as Parkinson's, epilepsy, neurosis, neurasthenia and neuralgia, in a perspective of biomedicine and informatics. Error could happen when we calculate EFI and ignore minor magnetic effects ${ }^{[13-14]}$.

\section{ACKNOWLEDGMENTS}

We thank Dr. Cheng, Yaoting, Mrs Li, Qiongqiu, Mr. Zou Zijia and Mrs. Zheng, Chaoying for financial support and Miss Vivien Cheng for helpful suggestions and comments, for this publication.

\section{REFERENCES}

1. Klafki, H.W., M. Staufenbiel, J. Kornhuber and J. Wiltfang, 2006. Therapeutic approaches to Alzheimer's disease. Brain, 129: 2840-55.

2. Bullock, R., 2004. Future directions in the treatment of Alzheimer's disease. Expert. Opin. Investig. Drugs, 13: 303-14.

3. Cummings, J.L., 2004. Alzheimer's disease. N. Engl. J. Med., 35: 56-67.

4. Forsyth, E. and P.D. Ritzline, 1998. An overview of the etiology, diagnosis and treatment of Alzheimer disease. Phys. Ther., 78: 1325-31.

5. Alzheimer, A., 1907. Uber eine eigenartige Erkrankung der Hirnrinde. Allgemeine Zeitschrift fuer Psychiatrie, 64: 146-8.

6. Hodges, J.R., 2006. Alzheimer's centennial legacy: Origins, landmarks and the current status of knowledge concerning cognitive aspects. Brain, 129: 2811-22.

7. Kraepelin, E., 1910. Psychiatrie: Ein Lehrbuch fur Studierende und Arzte. In: Handbook of Psychiatry. 8th Edmn. Kraepelin E., (Ed.) Barth, pp: 593-632.

8. Nussbaum, R.L. and C.E. Ellis, 2003. Alzheimer's disease and Parkinson's disease. N. Engl. J. Med., 348: 1356-64.

9. Cowburn, R.F., 2000. Neurotransmitter receptor-Gprotein-mediated signal transduction in Alzheimer's disease. In: Cerebral Signal Transduction: From First to Fourth Messengers. Reith M.E.A., (Ed.) Humana Press, pp: 129-149.

10. Masters, C.L. and K. Beyreuther, 2006. Alzheimer's centennial legacy: Prospects for rational therapeutic intervention targeting the Abeta amyloid pathway. Brain, 129: 2823-39. 
11. Apostolova, L.G., I.D. Dinov, R.A. Dutton, K.M. Hayashi, A.W. Toga, J.L. Cummings and P.M. Thompson, 2006. 3D comparison of hippocampal atrophy in amnestic mild cognitive impairment and Alzheimer's disease. Brain, 129: 2867-73.

12. Raskind, M.A., C.W. Wilkinson and E.R. Peskind, 2002. Aging and Alzheimer's disease. In: Hormones, Brain and Behavior. Plaff D.W., et al, (Ed.), Academic Press, pp: 637-44.

13. Cheng, K. and C.H. Zou, 2006. Electromagnetic field effect on separation of nucleotide sequences and unwinding of a double helix during dna replication. Med. Hypotheses, 66: 148-153.

14. Cheng, K. and C.H. Zou, 2006. Informatics and physics models of recognitions of DNA replication and their biomedical applications. Am. J. Appl. Sci., 3: 2059-62.

15. Martinoia, S. and P. Massobrio, 2004. ISFETneuron junction: circuit models and extracellular signal simulations. Biosens. Bioelectron., 19: 148796.

16. Kandel, E.R., J.H. Schwartz, T.M. Jessell, 2000. Principles of Neural Science. McGraw-Hill Medical. 4th Edn.

17. Cheng, K., P.P. Tarjan and C.H. Zou, 1993. Schrodinger equation, Maxwell-Bolzmann distribution and single channel current. Biom. Sci. and Instrumentation, 29: 361-367.

18. Cheng, K., 1997. Improved 3-D quantum mechanical models of ion movements in a cylindrical ion-channel. In: Proc. 16th Southern Biomed. Eng. Conf. J.D. Bumgardner and A.D. Puckett, (Ed.) IEEE, pp: 220-223.

19. Marshall, J.L., 1965. Introduction to Signal Theory. Scranton (Pennsylvania). International Text Book Company, pp: 185-235.

20. Nacher, V., S. Ojeda, C. Cadarso-Suarez, J. RocaPardinas and C. Acuna, 2006. Neural correlates of memory retrieval in the prefrontal cortex. Eur J. Neurosci., 24: 925-36.
21. Buckner, R.L. and W. Koutstaal, 1998. Functional neuroimaging studies of encoding, priming and explicit memory retrieval. Proc. Natl. Acad. Sci. U. S. A., 95: 891-8.

22. Soul'e, J., E. Messaoudiand and C. R. Bramham, 2006. Brain derived neurotrophic factor and control of synaptic consolidation in the adult brain. Biochem. Soc. Trans., 34: 600-4.

23. Wang, H., Y. Hu and J.Z. Tsien, 2006. Molecular and systems mechanisms of memory consolidation and storage. Prog. Neurobiol., 79: 123-35.

24. Fukazawa, Y., Y. Saitoh, F. Ozawa, Y. Ohta, K. Mizuno, K. Inokuchi, 2003. Hippocampal LTP is accompanied by enhanced F-actin content within the dendritic spine that is essential for late LTP maintenance in vivo. Neuron, 38: 447-60.

25. Lyford, G.L., K. Yamagata, W.E. Kaufmann, C.A. Barnes, L.K. Sanders, N.G. Copeland, D.J. Gilbert, N.A. Jenkins, A.A. Lanahan and P.F. Worley, 1995. Arc, a growth factor and activity-regulated gene, encodes a novel cytoskeleton-associated protein that is enriched in neuronal dendrites. Neuron, 14: 433-45.

26. Link, W., U. Konietzko, G. Kauselmann, M. Krug, B. Schwanke, U. Frey and D. Kuhl, 1995. Somatodendritic expression of an immediate early gene is regulated by synaptic activity. Proc. Natl. Acad. Sci., U. S. A., 92: 5734 -8.

27. Matsuzaki, M., N. Honkura, G.C. Ellis-Davies and H. Kasai, 2004. Structural basis of long-term potentiation in single dendritic spines. Nature, 429: 761-6.

28. Blum, R., A. Konnerth, 2005. Neurotrophinmediated rapid signaling in the central nervous system: mechanisms and functions. Physiology (Bethesda), 20: 70-8. 